\title{
LXVIII. Researches in the undulatory theory of light continued: On the absorption of light
}

\author{
John Tovey Esq.
}

To cite this article: John Tovey Esq. (1839) LXVIII. Researches in the undulatory theory of light continued: On the absorption of light, Philosophical Magazine Series 3, 15:98, 450-455, DOI: $10.1080 / 14786443908649921$

To link to this article: http://dx.doi.org/10.1080/14786443908649921

册 Published online: 01 Jun 2009.

Submit your article to this journal $\widetilde{x}$

Џ Article views: 3

Q View related articles $\square$ 


\section{[ 450 ]}

LXVIII. Researches in the Undulatory Theory of Light continued: On the Absorption of Light. By JoHN 'Tovey, Esg.

To the Editors of the Philosophical Magazine and Journal. Gentlemen,

A T the conclusion of my last paper (L. \& E. Phil. Mag. vol. A xiv. p. 32\%.) I said that I expected to be able to show that our formulæ were adequate to explain the cause of the absorption of light. This expectation was founded on the circumstance that the quantity which we have denoted by $k$, has, without any reason, been tacitly assumed to be entirely real; while it is obvious that, if $k$ be partly imaginary, the formula require to be transformed into others which will indicate an absorption depending on $k$ and $x$. The values of $k$ depend on the nature of the medium; and it is well known that every medium absorbs more or less of the light which falls on it. Hence we infer that these values cannot be entirely real. Indeed, if they were so, the formulæ which we have previously deduced would show that no absorption could take place.

To effect the required transformation of our formulae, I have had recourse to a method of investigation more general, and, I think, more easy, than any which we have previously used. This method I now proceed to develop; setting out from the differential equations, given at page 11. vol. xii. of your Journal, viz.

$\frac{d^{2} \eta}{d t^{2}}=m \Sigma\{\phi(r) \Delta \eta+\psi(r)(\Delta y \Delta \eta+\Delta z \Delta \zeta) \Delta y\}$,
$\frac{d^{2} \zeta}{d t^{2}}=m \Sigma\{\phi(r) \Delta \zeta+\psi(r)(\Delta y \Delta \eta+\Delta z \Delta \zeta) \Delta z\}$.

In deducing these equations the masses of the molecules were supposed to be all equal*. When this is not the case, $m$, which denotes any one of these masses, must be placed under the sign, $\Sigma$, of summation. If for the sake of abridgement, we put

the equations (1.) will become

$$
\begin{aligned}
& m\left\{\phi(r)+\psi(r) \Delta y^{2}\right\}=p, \\
& m\left\{\Phi(r)+\psi(r) \Delta z^{2}\right\}=p^{\prime},
\end{aligned}
$$

$$
\begin{aligned}
& \frac{d^{9} \eta}{d t^{2}}=\Sigma(p \Delta \eta+q \Delta \zeta), \\
& \frac{d^{9} \zeta}{d t^{2}}=\Sigma\left(p^{\prime} \Delta \zeta+q \Delta \eta\right) ;
\end{aligned}
$$

which are true whether the masses are equal or unequal.

* See L. \&:E. Phil. Mag. vol. viii. p. 7-9. 
Since we suppose $x$ to be normal to the wave surface, and, consequently, the general expressions for the displacements $\eta$ and $\zeta$ to be functions of $x$ and $t$, we may conceive each of these expressions to be developed in a series of the powers and products of $e^{x}$ and $e^{t}$ : where $e$ is the number whose hyperbolical logarithm is unity. Every term of the developments will be of the form $\alpha e^{\nu t+\kappa x}$ : where $\alpha, v, x$, are constant quantities.

$$
\text { Suppose } \quad \eta=\alpha e^{\nu t+\kappa x}, \zeta=\rho \alpha e^{\nu t+\kappa x},
$$

and put we shall then find

$$
\begin{aligned}
& \frac{d^{2} \eta}{d t^{2}}=v^{2} \alpha \omega, \quad \frac{d^{2} \zeta}{d t^{2}}=\nu^{2} \rho \alpha \omega, \\
& \Delta \eta=\left(e^{\kappa \Delta x}-1\right) a \omega, \quad \Delta \zeta=\left(e^{k \Delta x}-1\right) \rho \alpha \omega . \\
& \text { Put } \quad \Sigma p\left(e^{k \Delta x}-1\right)=s \text {, } \\
& \Sigma p^{\prime}\left(e^{\kappa \Delta x}-1\right)=s^{\prime} \text {, } \\
& \Sigma q\left(e^{\kappa \Delta x}-1\right)=s_{l},
\end{aligned}
$$

and substitute the values (6.) and (7.) in (3.); we shall then have, on omitting the common factor $\alpha$ w,

$$
\begin{aligned}
& \nu^{2}=s+\rho s_{l}, \\
& \nu^{2}=s^{\prime}+\frac{s,}{\rho} .
\end{aligned}
$$

Hence it appears that, when the arbitrary quantities $v, \rho, x$, are made to satisfy these two conditions, the expressions (4.) are a particular solution of the equations (3.). Now, since these equations are of the first degree, they may be satisfied, not only by the expressions (4.), but by the sums of any number of expressions of the same form; therefore we may put

$$
\eta=\Sigma \alpha e^{\nu t+\kappa x}, \quad \zeta=\Sigma \rho \alpha e^{\nu t+\kappa x} ;
$$

which will represent the general values of $\eta$ and $\zeta$, developed, as we have previously supposed, in series of the powers and products of $e^{t}$ and $e^{x}$.

It is obvious, by (10.), that the values of $\nu$ must be entirely imaginary; for, if not, the displacements, $\eta$ and $\zeta$, would contain terms increasing indefinitely with the time; which they cannot do, because the motions under consideration are, by hypothesis, merely vibratory. We may therefore put

$$
\begin{aligned}
\nu= & n \sqrt{-1}, \\
& 2 \mathrm{G} 2
\end{aligned}
$$


$452 \mathrm{Mr}$. Tovey's Researches in the Undulatory Theory:

and change, accordingly, the equations (9.) into

$$
\begin{aligned}
n^{2}+s+\rho s_{l} & =0, \\
n^{2}+s^{\prime}+\frac{s_{1}}{\rho} & =0 .
\end{aligned}
$$

By eliminating $\rho$ from (12.) we have

$$
\left(n^{\mathrm{o}}+s\right)\left(n^{2}+s^{\prime}\right)=s_{i}^{2} \text {. }
$$

The sums $s, s^{\prime}, s_{s}$, comprise only $\varkappa$ and quantities depending on the nature of the medium; therefore, the last equation determines the value of $x$, supposing that of $n$ to be given.

Whatever be the nature of $x$, we may, by the principles of analysis, suppose $\quad x=h(\cos i+\sqrt{-1} \sin i)$ : where $h$ and $i$ are real quantities. Therefore, since

and

$$
e^{\kappa \Delta x}=1+\frac{x \Delta x}{1}+\frac{x^{2} \Delta x^{2}}{1 \cdot 2}+\frac{x^{3} \Delta x^{3}}{1.2 .3 .}+\& c .
$$

$(\cos i+\sqrt{-1} \cdot \sin i)^{m}=\cos m i=\sqrt{-1} \cdot \sin m i$, (where $m$ is any number,) we have

$$
\begin{aligned}
& e^{\kappa} \Delta x-1=\frac{h \cos i}{1} \Delta x+\frac{h^{2} \cos 2 i}{1.2} \Delta x^{2}+\& \mathrm{c} . \\
& +\sqrt{-1} \cdot\left(\frac{h \sin i}{1} \Delta x+\frac{h^{2} \sin 2 i}{1.2} \Delta x^{2}+\& \mathrm{c} .\right) .
\end{aligned}
$$

By substituting this development of $\left(e^{\kappa \Delta x}-1\right)$ in the equations (8.), and, for the sake of abridgement, putting

$$
\begin{aligned}
& \mathrm{A}=\Sigma p \Delta x, \mathrm{~A}^{\prime}=\Sigma p^{\prime} \Delta x, \\
& \mathrm{~A}_{1}=\frac{\sum p \Delta x^{2}}{2}, \mathrm{~A}_{1}^{\prime}=\frac{\sum p^{\prime} \Delta x^{2}}{2}, \\
& \mathrm{~A}_{2}=\frac{\sum p \Delta x^{3}}{2 \cdot 3}, \mathrm{~A}_{2}^{\prime}=\frac{\sum p^{\prime} \Delta x^{3}}{2 \cdot 3}, \\
& \text { \&c. } \quad \mathrm{Bc.}=\Sigma q \Delta x, \\
& \mathrm{~B}_{1}=\frac{\sum q \Delta x^{2}}{2}, \\
& \mathrm{~B}_{2}=\frac{\sum q \Delta x^{3}}{2 \cdot 3},
\end{aligned}
$$

and $\sigma=\Lambda h \cos i+\mathbf{A}_{1} h^{2} \cos 2 i+\mathbf{A}_{2} h^{3} \cos 3 i+8 \mathrm{c}$.

$\sigma^{\prime}=\mathbf{A}^{\prime} h \cos i+\mathbf{A}_{1}^{\prime} h^{2} \cos 2 i+\mathbf{A}_{2}^{\prime} h^{3} \cos 3 i+\& \mathrm{c}$.

$\sigma_{1}=\mathbf{A} h \sin i+\mathbf{A}, h^{2} \sin 2 i+\mathbf{A}_{2} h^{3} \sin 3 i+\& \mathrm{c}$.

$\sigma_{1}^{\prime}=\mathrm{A}^{\prime} h \sin i+\mathrm{A}_{1}^{\prime} h^{2} \sin 2 i+\mathrm{A}_{2}^{\prime} h^{3} \sin 3 i+8 \mathrm{c}$. 
we find

$$
\begin{aligned}
& \sigma_{2}=\mathrm{B} h \cos i+\mathrm{B} h^{2} \cos 2 i+\mathrm{B}_{2} h^{3} \cos 3 i+\& \mathrm{c} . \\
& \sigma_{3}=\mathrm{B} h \sin i+\mathrm{B}_{1} h^{2} \sin 2 i+\mathrm{B}_{2} h^{3} \sin 3 i+\& \mathrm{c} .
\end{aligned}
$$

$$
s=\sigma+\sqrt{\overline{-1}} \cdot \sigma_{1}, \quad \underline{s^{\prime}=\sigma^{\prime}+\sqrt{-1} \cdot \sigma_{\prime}^{\prime},},
$$

Now suppose

$$
p=\beta(\cos \gamma+\sqrt{-1} \cdot \sin \gamma),
$$

and substitute the values of $s, s^{\prime}, s_{1}$, and $\rho$, in (12.); then, since $n$ is entirely real, we shall have

$$
\begin{aligned}
n^{2}+\sigma+\beta\left(\sigma_{2} \cos \gamma-\sigma_{3} \sin \gamma\right) & =0, \\
n^{2}+\sigma^{\prime}+\frac{1}{\beta}\left(\sigma_{2} \cos \gamma+\sigma_{3} \sin \gamma\right) & =0, \\
\sigma_{1}+\beta\left(\sigma_{2} \sin \gamma+\sigma_{3} \cos \gamma\right) & =0, \\
\sigma^{\prime}-\frac{1}{\beta}\left(\sigma_{2} \sin \gamma-\sigma_{3} \cos \gamma\right) & =0 .
\end{aligned}
$$

The four equations (22.) determine the values of $h, i, \beta, \gamma$, corresponding to any given value of $n$; and it may easily be seen that these equations will not be affected if we change the sign of $n$, or if we change, simultaneously, the signs of $i$ and $\gamma$. Therefore, since the general values of $\eta$ and $\zeta$ are the sums of all their particular values, we may give to $v, x, \rho$, in (10.), not only the values (11.), (14.), (21.), but also those which result from them, by changing the signs of $n, i, \gamma$. If we do so, and, for the sake of abridgement, put

$$
\begin{gathered}
h \cos i=\varepsilon, \quad h \sin i=k, \\
\beta(\cos \gamma+\sqrt{-1} \cdot \sin \gamma)=\rho_{1}, \\
\beta(\cos \gamma-\sqrt{-1} \cdot \sin \gamma)=\rho_{2},
\end{gathered}
$$

we shall have

$$
\begin{aligned}
& \eta=\Sigma\left\{e^{\epsilon x}\left(\alpha_{1} e^{(n t+k x)} \sqrt{-1}+\alpha_{2} e^{-(n t+k x) \sqrt{-1})}\right\}\right. \\
& \zeta=\Sigma\left\{e^{\epsilon x}\left(\rho, \alpha, e^{(n t+k x)} \sqrt{-1}+\rho_{2} \alpha_{2} e^{-(n t+k x) \sqrt{-1})}\right\}\right.
\end{aligned}
$$

where $\alpha_{1} \alpha_{2}$, are values of $\alpha_{3}$ and are entirely arbitrary. find

By changing the last expressions into circular functions, we

$$
\begin{aligned}
& \eta=\Sigma\left\{a e^{\epsilon x} \sin (n t+k x+b)\right\}, \\
& \zeta=\Sigma\left\{a^{\prime} e^{\epsilon x} \sin \left(n t+k x+b^{\prime}\right)\right\}:
\end{aligned}
$$

where $a, b, a^{\prime}, b^{\prime}$, are determined by the equations

$$
\begin{gathered}
a \sin b=\alpha_{1}+\alpha_{2}, \quad a \cos b=\left(\alpha_{1}-\alpha_{2}\right) \sqrt{-1}, \\
a^{\prime} \sin b^{\prime}=\rho_{1} \alpha_{1}+\rho_{2} \alpha_{2}, \quad a^{\prime} \cos b^{\prime}=\left(\rho_{1} \alpha_{1}-\rho_{2} \alpha_{2}\right) \sqrt{-1} .
\end{gathered}
$$


Since $\eta$ and $\zeta$ are entirely real, it appears, by (26.) and $(27$,$) , that \alpha_{1}$ and $\alpha_{2}$ must be of the forms

$$
\alpha_{1}=c_{1}+c_{2} \sqrt{-1}, \quad \alpha_{2}=c_{1}-c_{2} \sqrt{-1} \text { : }
$$

therefore, by (27.)

$$
a \sin b=2 c_{1}, \quad a \cos b=-2 c_{2} .
$$

Again, by (24.) and (27.),

$$
\begin{aligned}
a^{\prime} \sin b^{\prime} & =\beta(\cos \gamma+\sqrt{-1} \cdot \sin \gamma) \alpha_{1} \\
& +\beta(\cos \gamma-\sqrt{-1} \cdot \sin \gamma) \alpha_{2}, \\
a^{\prime} \cos b^{\prime} & =\beta(\cos \gamma+\sqrt{-1} \cdot \sin \gamma) \alpha_{1} \sqrt{=1} \\
& +\beta(\cos \gamma-\sqrt{-1} \cdot \sin \gamma) \alpha_{2} \sqrt{-1}
\end{aligned}
$$

hence, by (28.),

$$
\begin{aligned}
& a^{\prime} \sin b^{\prime}=2 \beta\left(c_{1} \cos \gamma-c_{2} \sin \gamma\right), \\
& a^{\prime} \cos b^{\prime}=-2 \beta\left(c_{2} \cos \gamma+c_{1} \sin \gamma\right) ;
\end{aligned}
$$

and hence, by (29.)

$$
\begin{array}{ll} 
& a^{\prime} \sin b^{\prime}=a \beta \sin (b+\gamma), \\
& a^{\prime} \cos b^{\prime}=a \beta \cos (b+\gamma) ; \\
\text { therefore } & a^{\prime}=a \beta, \quad b=b+\gamma, \\
\text { or } & a^{\prime}=-a \beta, \quad b=b+\gamma+\pi .
\end{array}
$$

Since $\alpha_{1}$ and $\alpha_{2}$ are arbitrary so also are $c_{1}$ and $c_{2}$, by (28.); and consequently $\alpha$ and $b$, by (25.).

By (23.) and (26.) we have

$$
\begin{aligned}
& \eta=\Sigma\left\{a e^{e x} \sin (n t+k x+b)\right\}, \\
& \zeta=\Sigma\left\{\beta a e^{e x} \sin (n t+k x+b+\gamma)\right\} .
\end{aligned}
$$

In these expressions, which are equally general with the formule (10.), all the symbols denote real quantities.

We have remarked that the equations (22.) determine the values of $h, i, \beta, \gamma$, for any given value of $n$. The values of $h, i$, determine those of $\varepsilon, k$, by (23.). Now the intensity of the light depends on $a e^{\epsilon x}$ and $\beta a e^{\epsilon x}$, so that if the vibrations in the ray under consideration be represented by (35.), its intensity will be expressed by

$$
\Sigma\left\{\left(a e^{\epsilon x}\right)^{2}+\left(\beta a e^{\epsilon x}\right)^{2}\right\} \text {. }
$$

(See Airy's Tract on Light, art. 23, and note.) Hence if we suppose the origin of $x$ to be at the surface of any medium on which a ray of light falls, we perceive that, unless $\varepsilon$ be zero, the intensity of the light will vary with $x$; and since experience shows, that whatever be the medium which a ray of light traverses, its intensity is thereby diminished, we infer that $\approx$ must have sensible values, and that these values must be negative. 
Mr. Stark on the Sand-pipes in the Chalk near Norwich. 455

Now, by (23.), $\varepsilon$ is connected with $k$; hence it follows that the diminution of the intensity of the light, in traversing any medium, depends on the thickness of the medium and the length of the wave.

This inference agrees with what we learn by experiment; therefore-we may say that our formulæ afford, so far, an explanation of the cause of the absorption of light. The cause subsists in the values of $x$ given by the equation (13.). In all media for which these values are, severally, partly real and partly imaginary, absorption must take place.

It is my intention to pursue this subject further in another paper.

$$
\begin{aligned}
& \text { I am, Gentlemen, yours, \&c., } \\
& \text { Loun Tover. }
\end{aligned}
$$

LXIX. Observations on Mr. Lyell's Paper, entitled, "On the tubular Cavities filled with Gravel and Sand, called Sandpipes, in the Chalk near Norwich." By W'rlliam Stank, F.G.S.

To the Editors of the Philosophical Magazine and Journai. Dear Sirs,

YOUR Magazine of the present month contains a paper by $\mathrm{Mr}$. Lyell on the "Sand-pipes in the chalk near Norwich," upon which I beg to offer a few remarks.

In the first place allow me to observe that the description which Mr. Lyell has given of the forms of the chalk pits and sand-pipes in this neighbourhood is very accurate, and the engravings by which it is illustrated are excellent representations of the originals. The hypothesis, however, that he advances relative to the origin of their formation, is, in my opinion, far from being satisfactory. The order of arrangement which he has adopted does not appear to me the most suitable : nevertheless I will take it as it stands.

He says, "For the distance of several inches, or even in some places four or five feet from its junction with the sandpipes, the chalk at Eaton is moist and softened, and becomes friable when dried, and is discoloured by containing a slight mixture of fine sand, clay and iron, the same chalk being quite pure, and perfectly soluble in acids at points more remote from the pipes."

This does not correspond with the result of my experiments upon the same chalk, however remote from the sandpipes. I did not find it "quite pure"; it was not "perfectly 